\title{
The contribution of imaging and digitised data to mandibular reconstruction and implant stabilised occlusal rehabilitation: a case report ${ }^{\dagger}$
}

\author{
R. M. Watson, ' T. J. Coward, ${ }^{2}$ R. K. F. Clark, ${ }^{3}$ and S. Grindrod, ${ }^{4}$
}

\begin{abstract}
Different methods are recommended for the surgical reconstruction of the resected mandible. The advantages for implant stabilised prostheses in restoring the occlusion are recognised but few papers provide adequate data to identify the successful outcome of treatment. The literature is reviewed and the advantages of imaging together with the use of digitised data is highlighted by a case requiring rehabilitation with enhanced planning methods.
\end{abstract}

$\mathrm{D}$ iscontinuity of the mandible, created by trauma, infection or the removal of a tumour results in cosmetic deformity, psychological impairment and functional disability. Previously the combination of surgical and prosthetic rehabilitation frequently required multiple operations and the use of a removable prostheses. This failed to provide effective mastication caused by the limitation of necessary jaw movements, difficulty in manipulation of the food bolus, and instability of the prostheses supported by reconstructed soft tissue. ${ }^{1}$

Mandibular reconstruction is therefore appropriate to stabilise the residual segments, to maintain the contours of the patient's face and restore masticatory function. The latter is more likely to be achieved by the use of carefully planned autogenous bone grafts and the implantation of reconstructed sites to allow the provision of an implant supported fixed prosthesis. ${ }^{2,3}$

${ }^{\dagger}$ Presented at the annual BSSPD Conference, Liverpool, UK, 1999

$1^{*}$ Professor of Dental Prosthetics, ${ }^{2}$ Chief Dental Instructor, ${ }^{3}$ Consultant, Part-time Senior Lecturer, GKT Dental Institute, King's College London; ${ }^{4}$ Clinical Scientist, University College London ${ }^{*}$ Correspondence to: Professor R. M. Watson, Head of Department, Department of Prosthetic Dentistry, GKT Dental Institute, King's College LondonDenmark Hill Site, Caldecot Road, London SE5 9RW email: roger.watson@kcl.ac.uk

REFEREED PAPER

Received 07.10.99; Accepted 29.09.00

(C) British Dental Journal 2001; 190: 296-300

\section{Alternative approaches to grafting}

The choice of grafting method is controversial in oral and maxillofacial surgery. Three methods have been advocated - autogenous corticocancellous block bone as a free graft ${ }^{4,5}$ or as a vascularised bone flap, ${ }^{6-8}$ or corticoncancellous bone chips may be packed in alloplastic trays. ${ }^{9,10}$ This controversy has been reviewed by Carlson and Marx ${ }^{11}$ and Wells. ${ }^{12}$ Free grafts are used more for bone enhancement as onlay grafts than for restoration of full thickness defects. Vascularised bone flaps have the disadvantage that there is no bone or part of a bone which is the same shape as the mandible, whereas an alloplastic tray can be custom made. ${ }^{13,14}$ Good success rates are claimed for both methods. The case described below contains both methods. The success of osseointegrated titanium implants is well

\section{In brief}

- Use of an appropriate software package enables computer assessment of the form/dimensions of jaws and facial dimensions

- The outcome of intended surgical and restorative changes can be accurately assessed offering a more radical approach to therapy

- Pre- and post-operative models can be prepared to visualise the outcome of maxillofacial rehabilitation documented with 5-year success rates in the order of $98 \%$ in the edentulous mandible. ${ }^{15}$ The success of implantation into mandibles with grafted bone is mainly anecdotal. Success rates of about $75 \%$ may be expected ${ }^{16}$ although higher figures have been recorded in two studies.

One retrospective 12-year longitudinal study of 31 patients, 23 of whom received free autogenous bone grafts recorded a cumulative survival rate of $93 \%$. More than $50 \%$ of the patients had functional implants longer than 5 years. ${ }^{17}$ Another study of nine patients recorded an $85 \%$ survival rate for 33 implants placed between 16 and 53 months. ${ }^{18}$

\section{Clinical case}

Presenting features and treatment of the patient

The female patient aged 53 had an inherited disorder, basal cell naevus syndrome (Gorlin's Syndrome), seen also in two siblings. She reported a series of operations on her jaws over a period of 25 years.

At 48 years of age she presented with an extensive cyst of the right mandible and bimaxillary cysts. The right mandible was resected and a free fibula autogenous vascular bone graft inserted to prevent pathological fracture (Figs 1,2).

The patient subsequently experienced difficulty in chewing caused by a restricted occlusion and there was a marked deviation of the chin to the left, producing noticeable facial asymmetry.

On examination intra-orally there was gross asymmetry of the jaws at rest with the need for the patient to move the mandible into an habituated occlusion (Fig. 3).

The rest position was recorded where the mandible was aligned without occlusal displacement and the dental casts were mounted to allow: (i) the construction of a silicone support to enable CT scanning of 


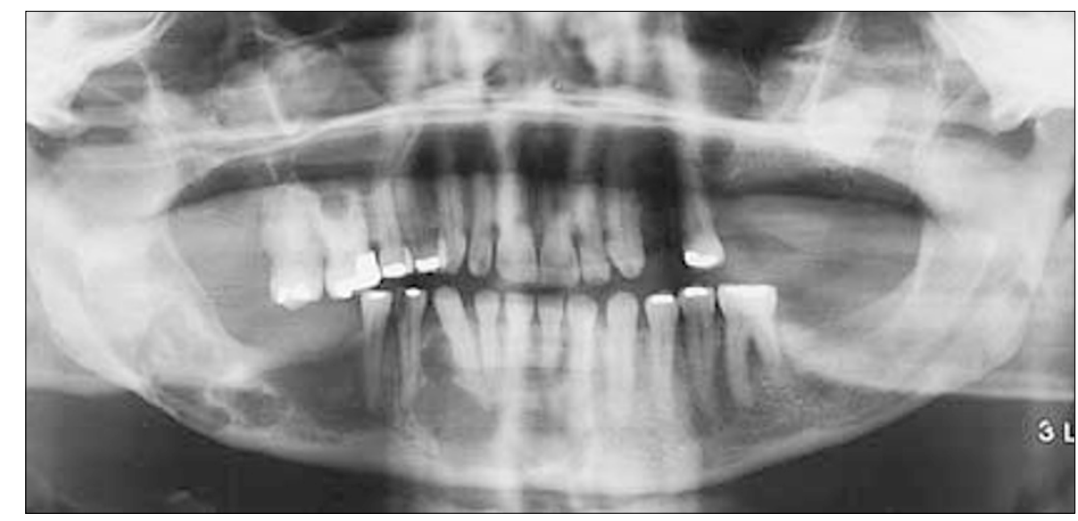

Fig. I x-ray of the jaws showing multiple cysts associated with Basal Cell Naevus Syndrome (Gorlin's Syndrome)

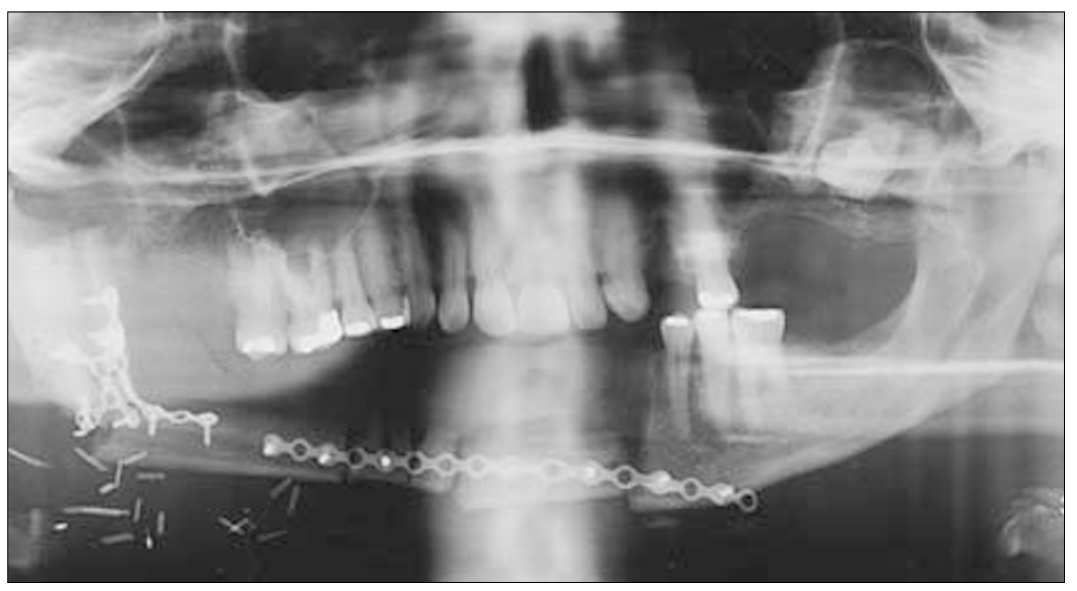

Fig. 2 -ray showing the mandible reconstructed with a vascularised fibula graft 2 years post operation
However, a 3-axis milling machine is only capable of producing the details of 3-surface forms in a model and it was necessary to merge the superior and inferior images of the mandible by using the custom software in order to create a 2-part mould. Thus the superior and lateral sides together with the inferior and lateral sides were represented in the mould within which a stone model was cast. The mould had to be sectioned further to release the cast of the mandible (Fig. 7). Thus both the computer image and model enabled the team to appreciate the problem and consider its solution.

The replica stone mandible was positioned against the polyurethane maxilla, confirming the occlusal discrepancy.

A measured $11 \mathrm{~mm}$ was cut from the stone mandible to align the left occlusion and position the right edentulous jaw below the maxillary arch. Next a wax extension was added to the stone jaw, to create an the jaws using a Siemens Somatron Helical Scanner and (ii) a visual assessment of the discrepancy between the jaws.

The data from the scan was displayed as a $3 \mathrm{D}$ image on the computer screen using a customised software program developed by University College London to show the mandibular rest position including the silicone support, and then with this support extracted from the image by the computer to show the discrepancy more clearly (Fig. 4).

The graft had restored the inferior border of the mandible but the dimensions, assessed as $15 \mathrm{~mm}$ in width and $7 \mathrm{~mm}$ in thickness, were insufficient to produce sound implant anchorage (Fig. 5).

In order to align the left occlusion of the residual arch and the right mandible with the maxilla, computer analysis showed that a segment of the graft measuring $11 \mathrm{~mm}$ required to be subtracted from the image (Fig. 6).

With this planning completed, data from the scan showing the original image was downloaded to a Bridgeport numerically controlled 3-axis milling machine that produced a positive model of the maxilla and skull base together with the cervical spine.

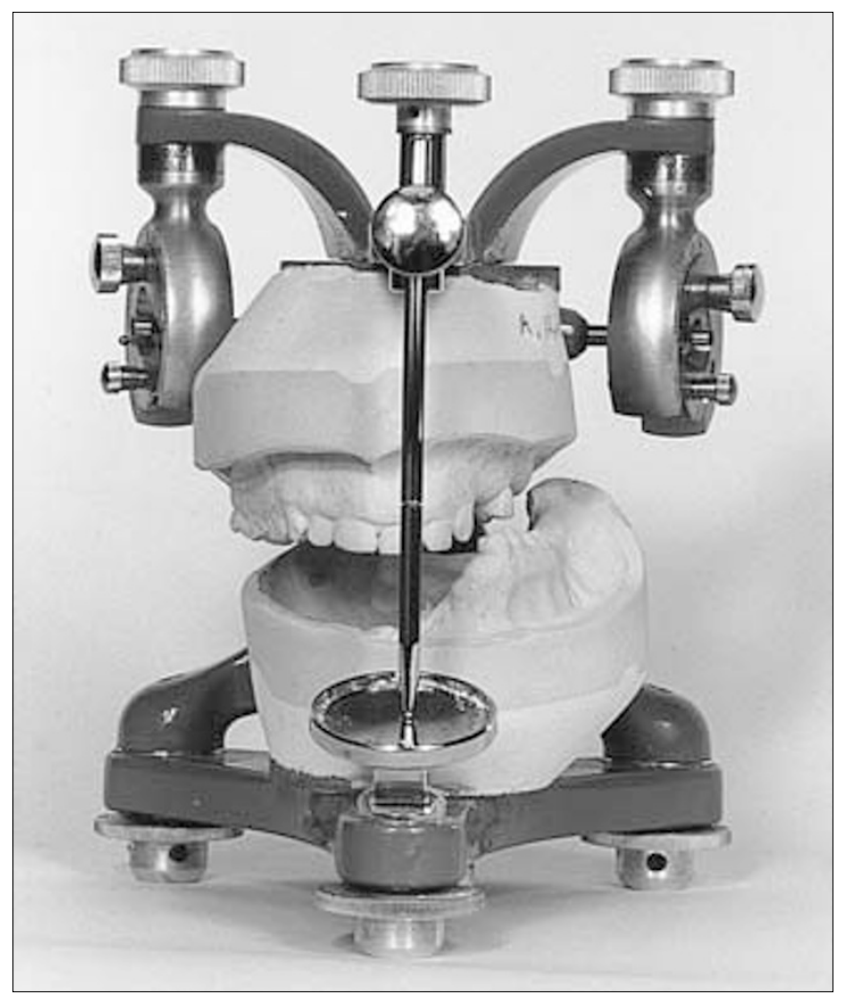

Fig. 3 Casts of the maxilla and mandible articulated to show the horizontal asymmetry in the resting jaw posture. The position has been recorded with silicone putty. The stent was used for recording the $C T$ scan 


\section{implants}

Fig. 4 Asymmetry of the jaws in the computer image, created from data downloaded from the CT scan. Note the obvious potential cross-bite of the left sides of the dental arches

Fig. 6 Realignment of the mandible by removing an II $\mathrm{mm}$ segment in the graft adjacent to the residual mandible, allows left sided occlusal contact of the residual dental arches. The right side of the reconstructed jaws are now positioned in alignment suitable to reconstruct the occlusion

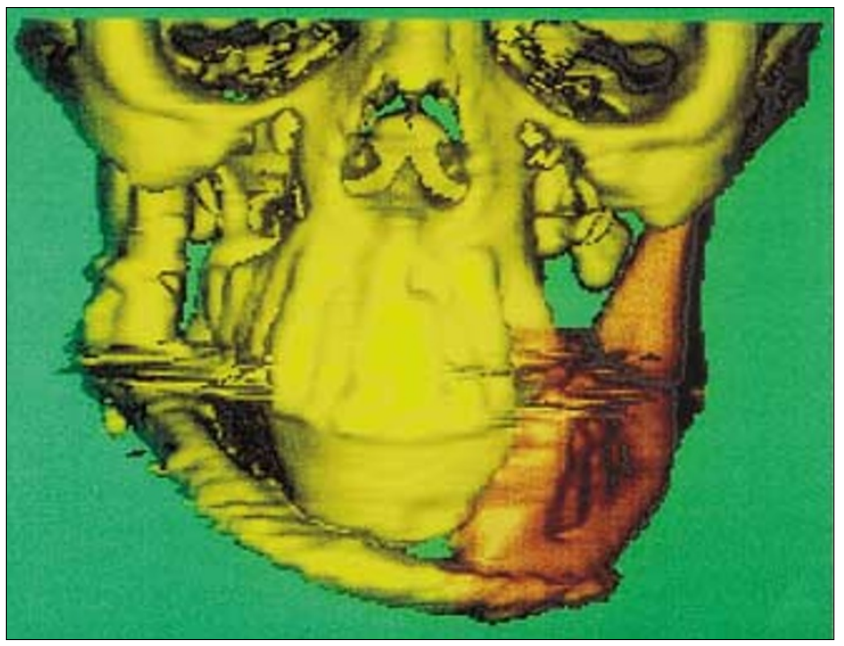

appropriate ridge to be enclosed by a titaum mesh tray.

Using the method employed by Samman et al. ${ }^{14}$ a duplicate was made in wax of the revised mandibular body extending from in front of the right angle to the left premolar. The wax replica jaw was used to prepare a 2-part acrylic mould on which titanium mesh was first swaged on the inferior surface to create a fitting tray. Then additional mesh was swaged over the second mould which provided the superior surface and around the sides of the lower tray to produce a lid (Fig. 8).

With an extra-oral approach the right grafted mandible was exposed surgically and the measured segment cut from the jaw, close to the midline adjacent to the residual mandible. The inferior titanium tray was screwed to the jaw to unite the mandible and cortico-cancellous grafts were removed from the iliac crest. The lid was positioned and the void completely filled with tightly packed cancellous material. After closing the wound with a drain, arch bars and eyelet wires were applied to secure the intended occlusion on the left side.

Almost 18 months later the case was worked up using a diagnostic wax-up for the insertion of titanium endosseous implants. Five self tapping $3.75 \mathrm{~mm}$ diameter Brånemark implants of 13 and $15 \mathrm{~mm}$ lengths were inserted into surprisingly dense bone, following removal of the lid (Fig. 9).

Two cover screws were lost in the
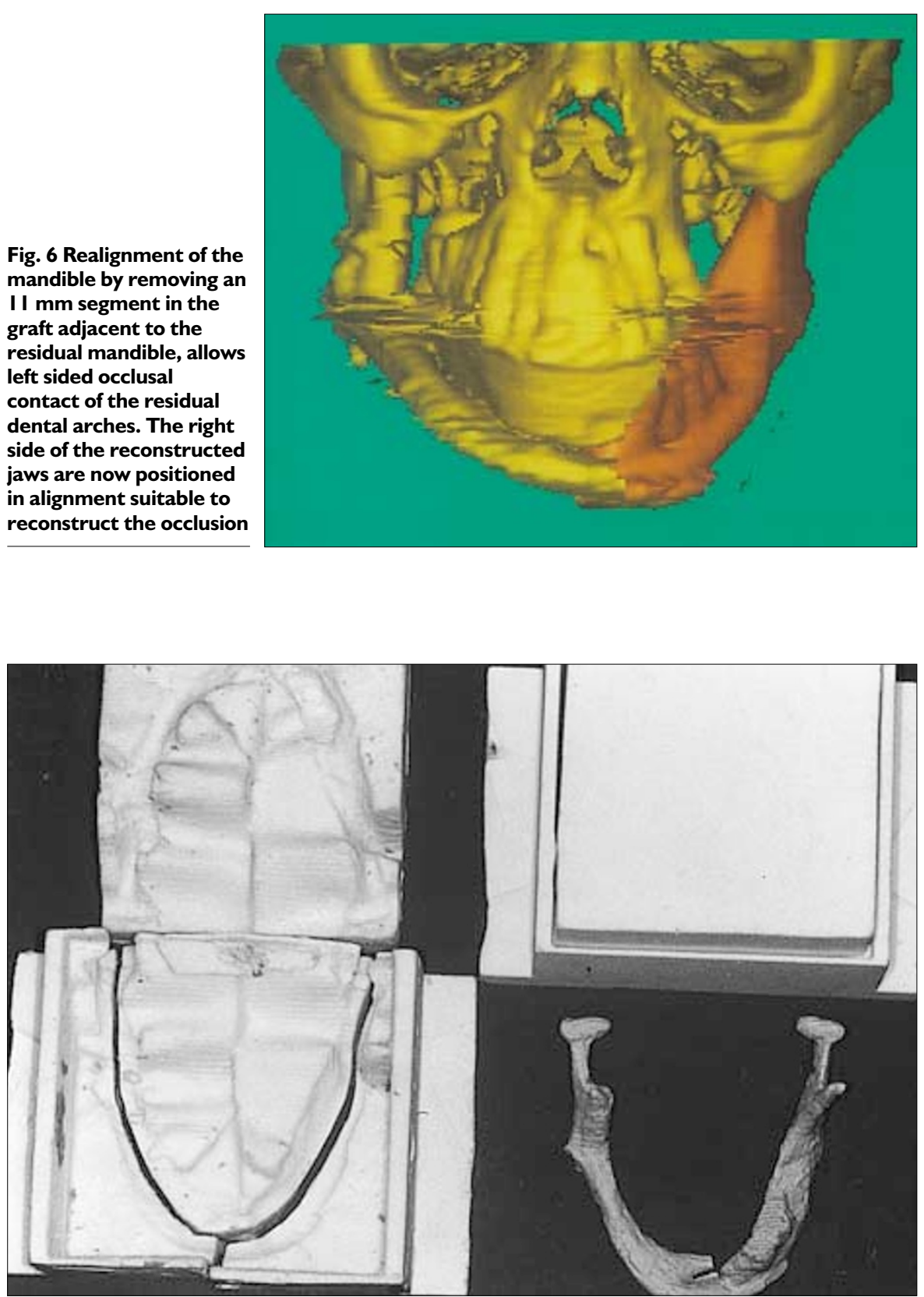

Fig. 7 The 2-part negative moulds created by using data from a custom software package downloaded to a 3-axis milling machine. A model poured into the mould reveals the shape of the over size mandible. The model was released by sectioning the mould 

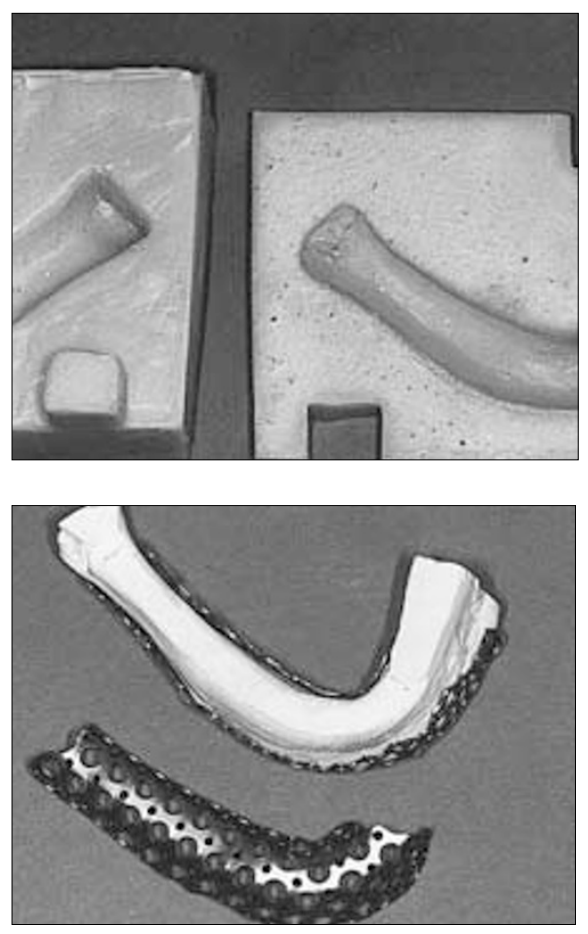

Fig. 8 Acrylic models constructed by duplication of the revised mandible, used as a die and counter-die in swaging the titanium mesh 2-part tray (previously described by Samman et al)

8-month interval before stage 2 when all implants were found to be well integrated.

A cast gold alloy frame with resin teeth was constructed as a fixed prosthesis using a combination of Mirus-Cone, Estheticone and standard abutments to support it. Considerable difficulty was encountered in registering a consistent jaw relationship, the patient not having worn a prosthesis for ten months.

Fortunately a reproducible jaw relation subsequently resulted in a cosmetic functional result. The health of the mucosal cuffs was good without evidence of inflammation but follow-up treatment was required to reinforce plaque control and replace one artificial tooth which fractured (Figs 10, 11).

\section{Discussion}

Restoration of a full thickness mandibular defect not only requires discontinuity of the mandible to be restored with a graft of sufficient length to restore symmetry but the lower border of the mandible must be cor-

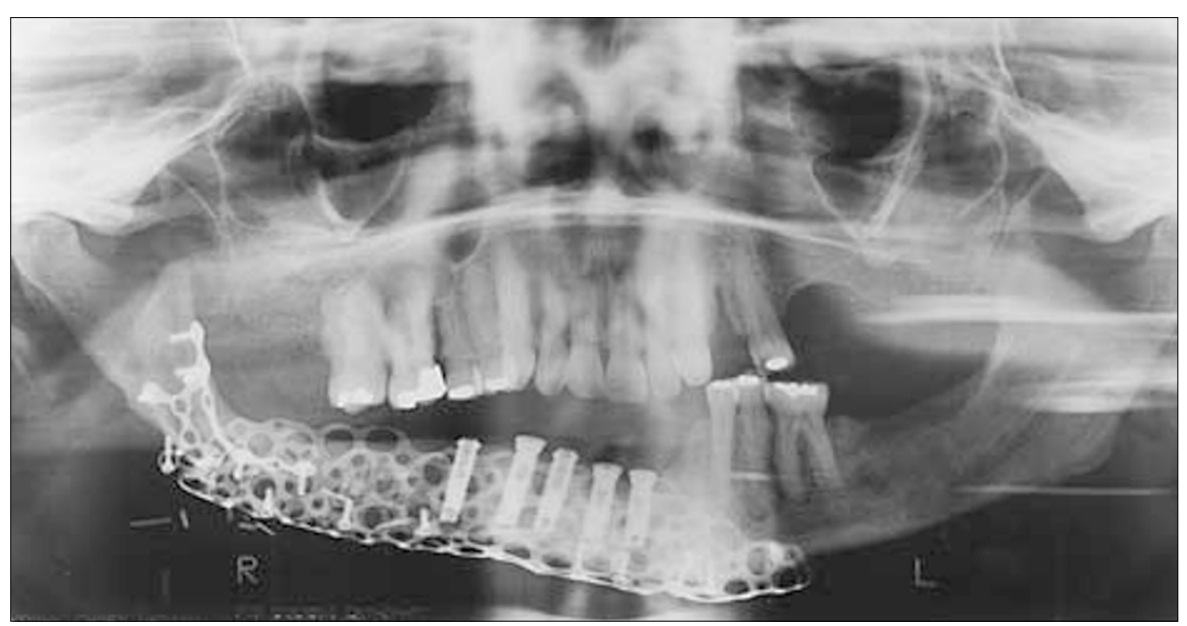

Fig. 9 Radiograph of the revised mandible following dental implantation

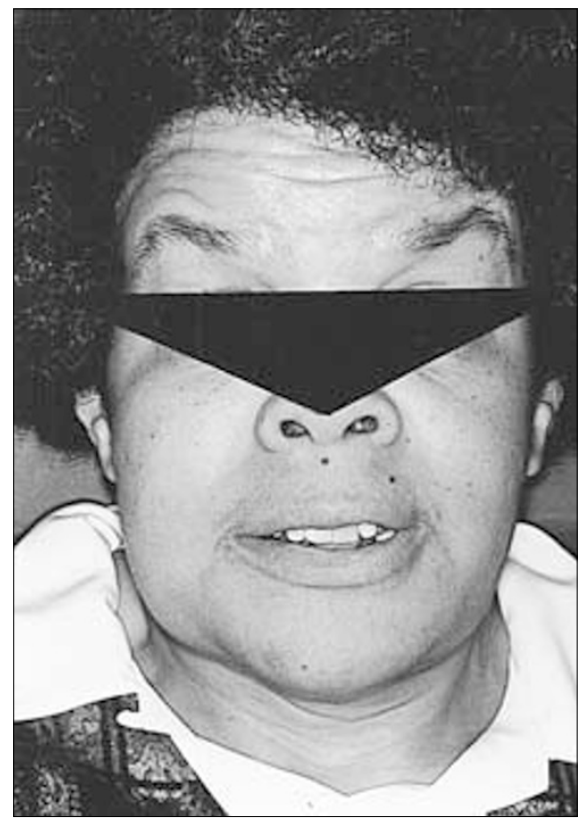

Fig. I0 Full page view of patient after treatment

rectly shaped to restore the patient's appearance. Whereas the intra-oral contours may be restored by onlay bone grafting, guides to the shape of the lower border are few especially when the defect crosses the midline.

Previously, in order to overcome the problem of restoring as closely as possible the external facial contours, Clark et al., ${ }^{13}$ Samman et al. ${ }^{14}$ and Tideman et al. ${ }^{10}$ have selected mandibles from a collection of skulls and then chosen one which most closely resembled the dimensions of the patient's and resected jaw. This mandible was duplicated in wax.

The dento-alveolar section that equated to the patient's residual natural dentition was removed from the wax model and replaced by the equivalent part of the patient's dental cast.

The replica jaw was then articulated. The wax was sculpted in the site of the resected mandible to produce the ideal form for future dental implant insertion.

The final step was to duplicate this intended shape in order to provide an acrylic model on which titanium mesh could be swaged in the form of a tray. The tray which held the bone graft that would restore continuity to the resected jaw, required adaptation to the cut ends of the mandible during the operation.

Now, CT imaging has recognised advantages not only in planning the surgical approach but also in achieving efficient

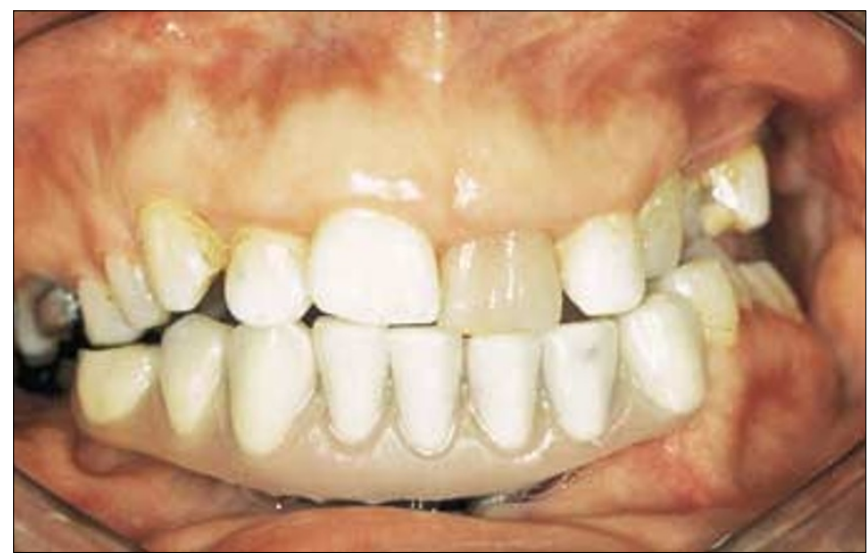

Fig. I I Alignment of the restored arch with the maxilla at the trial insertion of the fixed prosthesis 

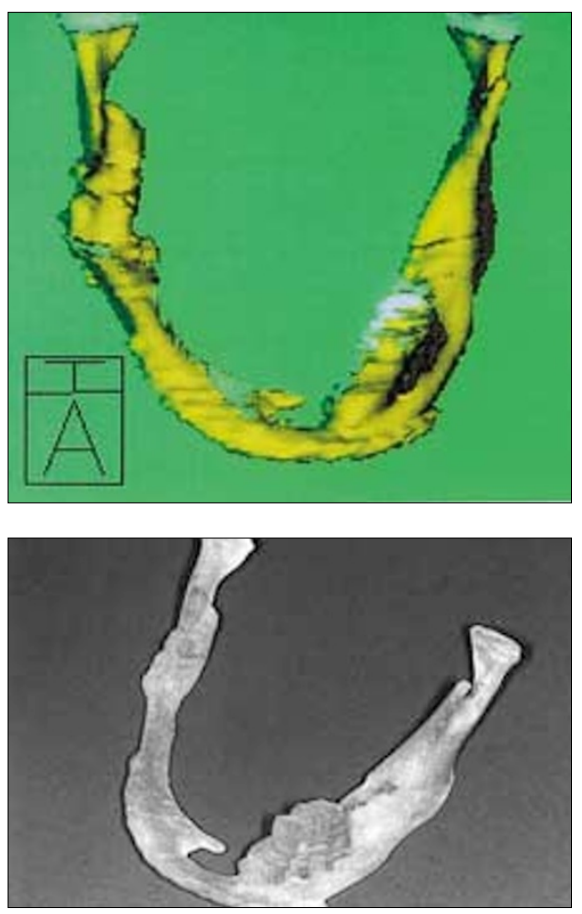

Fig. 12 Comparison of the computed image with a stereolithographic model of the grafted jaw before revision

immediate reconstruction. In this case the data from the scan downloaded to the computer allowed correction of an already reconstructed grafted mandible. However it is evident that other modifications could have been made to the image and so have produced the overall intended shape for the resected jaw, which envisaged the volume of bone needed to support dental implants and restore the external facial contours. This technique would have used a process of mirror imaging the untreated part of the mandible and siting the appropriate volume in the defective side (similar techniques have been described for prosthetic replacement of soft tissues). ${ }^{19}$ Data from the new image would then be available for creating a new model of the revised form of the jaw.

There is another refinement. In place of crude milling it is possible to create a more accurate model by downloading data for rapid process modelling with stereolithography. An example of this is seen in Figure 12 where information from the scan has produced a good replica of the patient's jaw in resin. With more modelling on the computer it would be possible to create a resin model of desired shape, including the reconstructed alveolus and so allow early preparation of the necessary die and counterdie for swaging the titanium tray. This is a more expensive option.

\section{Conclusion}

Where the mandible must be resected resulting in discontinuity, primary reconstruction is now the recommended treatment. The processes of pre-operative CT scanning and computer modelling provide the advantage of creating a well shaped titanium tray for autogenous non-vascular grafting, as well as rectifying architectural surgical problems arising from earlier treatment.

The authors wish to acknowledge the advice and surgical treatment of $\mathrm{Mr} G \mathrm{H}$ Forman, Emeritus Consultant Oral and Maxillofacial Surgeon and Miss C Bryant, Staff Grade Oral Surgeon.

1 Watson R M, Welfare R D, Islami A. The difficulties of prosthetic management of edentulous cases with hemi-mandibulectomy following cancer treatment. J Oral Rehabil 1984; 11: 201-214.

2 Tidstrom K D, Keller E E. Reconstruction of mandibular discontinuity with autogenous iliac bone graft: report of 34 consecutive patients. J Oral Maxillofac Surg 1990; 48: 336346.

3 Komisar A. The functional result of mandibular reconstruction. Laryngoscope 1990; 100: 364-374.

4 Afary W C. Reconstruction of mandibular discontinuity defects using antogenous grafting and a mandibular reconstruction plate. JOral Maxillofac Surg 1993; 51: 125-130. 5 Soderholm A L, Hallikainen D, Lindqvist C.
Radiologic follow-up of bone transplants to bridge mandibular continuity defects. Oral Surg 1992; 73: 253-261.

6 Hayter J P, Cawood J I. Oral rehabilitation with endosteal implants and free flaps. Int J Oral Maxillofacial Surg 1996; 25: 3-12.

7 Urken M L. Composite free flaps in mandibular reconstruction: a review of the literature. Arch Otolaryng Head Neck Surg 1991; 117: 724-32.

8 Vaughan E D. The radial forearm flap in orofacial reconstruction. Int J Oral Maxillofac Surg 1994; 23: 194-204.

9 Cheung L K, Samman N, Tang A C K, Tideman $\mathrm{H}$. Mandibular reconstruction with the dacron urethane tray; a radiologic assessment of bone re-modelling. J Oral Maxillofac Surg 1994; 52: 273-280.

10 Tideman H, Samman N, Cheung K K. Functional reconstruction of the mandible: a modified titanium mesh system. Int J Oral Maxillofac Surg 1998: 27:339-345.

11 Carlson E R, Marx R E. Mandibular reconstruction using cancellous cellular bone grafts. J Oral Maxillofac Surg 1996; 54: 889-897.

12 Wells M D. Mandibular reconstruction using vascularised bone grafts. J Oral Maxillfac Surg 1996; 54: 883-888.

13 Clark R K F, Chow T W, Chung W C, Luk H W $\mathrm{K}$, Samman N, Tideman H. A custom made titanium mesh tray for functional reconstruction of the mandible. Proc. BSSPD 1995 p8.

14 Samman N, Luk H W K, Chow T W, Cheung L K, Tideman H, Clark R K F. Custom made titaniuum mandibular reconstruction tray. Aust Dent J 1999; 44: 195-199.

15 Zarb G A, Schmitt A. The clinical effectiveness of osseo integrated dental implants; the Toronto study. Part 1 Surgical results. J Prosthet Dent 1990; 63: 451-457.

16 Hobkirk J A, Watson R M. Dental and maxillofacial implantology. London: MosbyWolfe, 1995; p11.

17 Keller F E, Tolman D, Eckert S. Endosseous implant and autogenous bone graft reconstruction of mandibular discontinuity: a 12-year longitudinal study of 31 patients. Int $J$ Oral Maxillofac Implants 1998; 13: 767-780.

18 Papageorge M B, Karabeton S M, Norris L H. Rehabilitation of patients with reconstructed mandibles using osseointegrated implants; Clinical report. Int J Oral Maxillofac Implants 1999; 14: 118-126.

19 Coward T J, Watson R M, Wilkinson I C. Fabrication of a wax ear by rapid process modelling using stereolithography. Int $J$ Prosthodont 1999: 12; 20 - 27. 